\title{
An assessment of the relative importance of body and plasma properties in determining the ion current distribution in the wake of an ionospheric satellite
}

\author{
URI SAMtR ${ }^{*}$ and Yoram J. KaUfman \\ *Space Physics Research Laboratory, University of Michigan, Ann Arbor, Michigan 48109, U.S.A. and tDepartment \\ of Geophysics and Planetary Sciences, Tel Aviv University, Israel
}

(Received 10 April 1979; in revised form 26 November 1979)

\begin{abstract}
Measurements of ion currents $\left(I_{+}\right)$, electron temperatures $\left(T_{e}\right)$, values of satellite potential $\left(\phi_{s}\right)$, ion concentrations $\left(N_{+}\right)$, and composition $\left(M_{+}\right)$, from the cylindrical electrostatic probe and the Bennett ion mass spectrometer on board the AE-C satellite were used in order to assess the degree and range of applicability of some simple theoretical wake models. The experimental results cover the plasma properties and parameter ranges:

$$
\begin{aligned}
& 1 \times 10^{4} \leq N_{+} \text {(ambient) } \leq 6.8 \times 10^{5} \mathrm{~cm}^{-3}, 760^{\circ} \mathrm{K} \leq T_{e} \leq 3135^{\circ} \mathrm{K}, \\
& 0.1 \leq\left|\phi_{\mathrm{s}}\right| \leq 1.3 \mathrm{~V}, 31 \leq \boldsymbol{R}_{\mathrm{D}}\left(\equiv \frac{\boldsymbol{R}_{\mathrm{u}}}{\lambda_{\mathrm{D}}}\right) \leq 216,2.6 \leq S_{\mathrm{AV}}\left\{=\frac{V_{s}}{\sqrt{\left[\frac{2 \mathrm{kT} T_{e}}{M_{+}(\text {average })}\right]}}\right\} \leq 9.0, \\
& 0.85 \leq\left|\phi_{N}\left(=\frac{e \phi_{s}}{k T_{e}}\right)\right|<20 \text {. }
\end{aligned}
$$

and the theoretical models are based on neutral and quasi-neutral approximations rather than on rigorous self-consistent solutions of the Vlasov-Poisson equations.

While the present investigation is subject to limitations, partly due to the above mentioned mathematical and physical simplifications and partly due to idealized body geometry, results were obtained which can be useful in the planning of space plasma physics experiments (in the area of flow interactions of supersonic and sub-Alfvenic plasmas with bodies) on board the spacelab/shuttle and/or any other large space platforms. The present investigation is an approximate theory-experiment comparison carried out in a parametric manner. We find that the relative $\mathrm{H}^{+}$concentration and electron temperature are very significant (compared with other plasma properties and parameters) in determining the ion distribution in the wake of an ionospheric satellite in quite a wide range of altitudes. We also find that for ionospheric plasmas where the $\mathrm{H}^{+}$concentration is in excess of $30 \%$, the ion distribution in the wake can 'satisfactorily' be described by a simple 'neutral approximation' model. This is in agreement with the theoretical predictions of AL'PERT (1976) and GUREvich et al. (1970). Furthermore, we find that a combined approximation which is a weighted sum of a neutral approximation for the $\mathrm{H}^{+}$contribution and a quasi-neutral approximation for the $\mathrm{O}^{+}$contribution gives better results than any of these approximations separately.
\end{abstract}

\section{INTRODUCTION}

While it is well known that the motion of a satellite in the ionosphere creates severe perturbations to its environment, the magnitude of such perturbations as a function of space and time is not yet quantitatively known. Neither are the strong perturbations in the wake zone behind the satellite understood in terms of fundamental plasma parameters and/or plasma properties.

Now that consideration is being given to the utilization of large space platforms, (e.g. Spacelab) as near-earth plasma laboratories where scientific questions of interest to space plasma physics can be studied in situ, the knowledge of the real signifcance of the perturbations created by space platforms to the neighboring plasma becomes of critical importance. It becomes important to know the variations in space and time of the perturbations as a function of plasma properties and (nondimensional) parameters. Hence being able to evaluate the quality and reliability of low-energy in situ measurements, performed by direct measurement devices.

In addition to the above 'application' of bodyplasma interaction studies, it is of great scientific interest to solar system plasma physics to investigate various aspects of the interaction of rarefied plasma with magnetized and non-magnetized bodies in a supersonic and sub-Alfvenic flow regime. Such experiments are planned for the Shuttle/Spacelab. Hence, studying the various aspects of body-plasma interactions is of interest to both science and technology. 
At the present time, before the shuttle/spacelab facility becomes available for scientific and technological rescarch, an attempt should be made to use selected data from available satellites and perform investigations (even partial in nature) that can contribute to our present knowledge and understanding regarding the distribution of ions and electrons around ionospheric satellites and in particular attempt to assess the variation of the latter with some body and plasma parameters. One of the important relevant questions in this context is that of the variation of the ion current depletion in the wake with body and plasma parameters. In fact, the ion current ratio $\gamma=\left[\frac{I_{+} \text {(wake) }}{I_{+} \text {(ambient) }}\right]$ can serve as a quantitative measure of the degree of disturbance the spacecraft motion has on its environmental spaceplasma. At the present time little is known experimentally about the variation of $\gamma$ with parameters such as:

$$
\begin{aligned}
R_{\mathrm{D}} & =\frac{R_{0}}{\lambda_{\mathrm{D}}}, \phi_{\mathrm{N}}=\frac{e \phi_{\mathrm{s}}}{\mathrm{k} T_{e}}, \\
S_{\mathrm{AV}} & =\frac{V_{\mathrm{s}}}{\sqrt{\left(\frac{2 \mathrm{k} T_{e}}{M_{+}(\mathrm{av})}\right)}} \text { and } \beta=\left(T_{e} / T_{+}\right), \text {where: }
\end{aligned}
$$

$R_{0}=$ satellite radius, $\lambda_{D}=$ ambient value of the Debye length, $\phi_{\mathrm{s}}=$ satellite potential, $\boldsymbol{T}_{c}=$ ambient electron temperature, $M_{+}(\mathrm{av})=$ average ionic mass, $v_{\mathrm{s}}=$ satellite velocity, $e=$ electronic charge, $\mathrm{k}=$ Boltzmann's constant.

Recently (SAmIR et al. 1979a) measurements of electron temperature from the cylindrical electrostatic probe (C.E.P.) and measurements of ion density and composition from the Bennett ion mass spectrometer (B.I.M.S.) both on the Atmosphere Explorer $\mathrm{C}(\mathrm{AE}-\mathrm{C})$ satellite were used to assess the influence of $T_{e}$ and $R=\frac{\left[N\left(\mathrm{O}^{+}\right)\right]}{\left[N\left(\mathrm{H}^{+}\right)\right]}, \quad$ [where: $N\left(\mathrm{O}^{+}\right)=$density of oxygen ions, $N\left(\mathrm{H}^{+}\right)=$density of hydrogen ions] on $\gamma$. The present paper is in a sense a continuation of the above investigation, attempting to present some information regarding the parametric and plasma properties interplay between some body and plasma parameters which determine $\gamma$, in the altitude range $410-980 \mathrm{~km}$. The results of the present study are expected to be useful in future planning of shuttle/spacelab missions and for testing the applicability of some theoretical wake models. Such studies should also be useful for future studies of flow interactions with test bodies in supersonic and sub-Alfvenic flow regimes. The latter is of interest to solar system plasma physics.

The objective of the present investigation are: (1) to examine experimentally the interplay between some plasma parameters and plasma properties in determining $\gamma,(2)$ to compare experimental results with some simplified theoretical models in order to assess their range of validity and applicability, (3) to provide information that will be useful to the planning of instrument location on ejectable ensembles of probes from the spacelab in future shuttle/spacelab missions, as well as to the planning of location of probes on the spacelab itself.

\section{MEASUREMENTS AND THEORETICAL. MODELS USED}

\subsection{Measurements}

The data used in the present investigation is based on electron temperature and ion-current measurements from the cylindrical electrostatic probe (C.E.P.) and measurements of ion density and composition from the Bennett ion mass spectrometer (B.I.M.S.), on the Atmospheric Explorer C (AE-C) satellite. The C.E.P. measurements (BRACE et al, 1973) were made at a distance of about $0.5 \boldsymbol{R}_{0}$ from the surface of the satellite $\left(R_{0}=\right.$ radius of the satellite) and the B.I.M.S. measurements were made at the closest vicinity to the satellite surface.

Information regarding the utilization of the above measurements in examining $I_{+}=f(\theta)$ and $\gamma=f\left\{T_{e} \frac{\left[N\left(\mathrm{O}^{+}\right)\right]}{\left[N\left(\mathrm{H}^{+}\right)\right]}\right\}$are given in SAMIR et al., (1979a, b). Here $\theta$ is the angle of attack and $I_{+}=$ion current. Detailed discussions on the C.E.P. and B.I.M.S. experiments are given in BRACE et al., (1973) and BRINTON et al., (1973) and will not be further discussed here.

The first four columns (from left) in Table 1 provide the altitude where the specific measurements were made, the relative hydrogen concentration, the satellite potential and the ratios $\gamma_{E X X}$. The $\mathrm{H}^{+}$concentration was obtained from the B.I.M.S. experiment and the spacecraft potential $\left(\phi_{s}\right)$ and the $\gamma_{\text {EXP }}$ values from the C.E.P. experiment. The $\phi_{s}$ was obtained in the conventional manner from the current-voltage characteristics of the probe (BRACE et al., 1973) and the $\gamma_{\mathrm{EXP}}$ values are the ratios of [ $I_{+}$(wake) $]:\left[I_{+}\right.$(ambient) $]$the latter being defined as

$$
I_{+}(\text {ambient })=I_{+}\left(\theta \sim 90^{\circ}\right)
$$

where: $\theta=$ angle of attack of the C.E.P. probe (SAmir et al., 1979a). The $I_{+}$(wake) is the ion 
Table 1. Ratio between experimental and theoretical results for some plasma properties and parameters $\rho^{k}=$ $[i(\theta) / i(\alpha)] /\left(n^{\mathrm{k}}(\theta) / n^{\mathrm{k}}(\alpha)\right]$

$(\mathrm{k}=n, q, c$ for each theory; $n=$ neutral approximation; $q=$ quasi-neutral approximation; $c=$ combined approximation.)

\begin{tabular}{|c|c|c|c|c|c|c|c|c|c|c|c|c|}
\hline Case No. & $\mathrm{H}(\mathrm{km})$ & $\mathrm{T}_{*}\left({ }^{\circ} \mathrm{k}\right)$ & $\left.W_{\mathbf{H}^{+}}\right)$ & $\phi_{s}(v)$ & $\gamma_{\text {EXP }}$ & $S_{\text {AV }}$ & $R_{\mathrm{D}}$ & $\phi_{N}$ & $\rho^{q}$ & $\rho^{n}$ & $\rho^{c}$ & $\gamma$ \\
\hline 1 & $276-313$ & 970 & 0. & -0.802 & 0.0078 & 8.04 & 135.7 & -9.4 & 26.0 & 30000 & 26 & 0.0003 \\
\hline 2 & 269 & 1425 & 0.0002127 & -0.1 & 0.02 & 6.59 & 251.75 & -0.82 & 17.1 & 228 & 16.2 & 0.00123 \\
\hline 3 & 312 & 2230 & 0.0004997 & -0.2 & 0.02 & 5.27 & 106.21 & -1.04 & 4.6 & 22 & 4.4 & 0.0045 \\
\hline 4 & 270 & 2470 & 0.000999 & -0.1 & 0.03 & 5.01 & 81.52 & -0.47 & 5,4 & 17.2 & 5. & 0.00597 \\
\hline 5 & 362 & 2175 & $"$ & -0.2 & 0.02 & 5.33 & 99.34 & -1.07 & 4.9 & 19 & 4.5 & 0.0044 \\
\hline 6 & 295 & 930 & $"$ & -1.3 & 0.02 & 8.16 & 133.24 & -16.25 & 80.5 & 103 & 45.8 & 0.00044 \\
\hline 7 & 308 & 1400 & $"$ & -0.1 & 0.02 & 6.65 & 160.86 & -0.83 & 18.0 & 63 & 14.4 & 0.00138 \\
\hline 8 & 375 & 1140 & " & 0.1 & 0.01 & 7.36 & 223.82 & 1.02 & 18.3 & 40 & 12.8 & 0.00078 \\
\hline 9 & $450-508$ & 948 & 0.00107 & -0.770 & 0.025 & 7.94 & 83.8 & -9.3 & 50.0 & 97 & 41 & 0.00052 \\
\hline 10 & $360-404$ & 977 & 0.00127 & -0.816 & 0.017 & 7.97 & 116.3 & -9.5 & 29.0 & 49 & 23 & 0.00055 \\
\hline 11 & 370 & 2375 & 0.00138 & -0.5 & 0.03 & 5.11 & 74.58 & -2.45 & 5.9 & 18.3 & 5.4 & 0.0056 \\
\hline 12 & $357-411$ & 1039 & 0.00140 & -0.771 & 0.0090 & 7.70 & 162.5 & -8.4 & 17.0 & 12 & 12 & 0.0007 \\
\hline 13 & 403 & 2029 & 0.00185 & -0.3 & 0.02 & 5.52 & 96.61 & -1.72 & 5,9 & 18 & 5.0 & 0.00403 \\
\hline 14 & 377 & 2660 & 0.00227 & -0.2 & 0.03 & 4.82 & 71.24 & -0.87 & 4.5 & 9.9 & 3.9 & 0.00767 \\
\hline 15 & 367 & 1280 & 0.00383 & -0.2 & 0.04 & 6.94 & 128.32 & -1.82 & 48.0 & 37.7 & 21.9 & 0.00183 \\
\hline 16 & 463 & 1200 & 0.00433 & -0.1 & 0.01 & 7.17 & 185.90 & -0.97 & 15.0 & 8.9 & 5.8 & 0.00172 \\
\hline 17 & 510 & 2560 & 0.0099 & -0.5 & 0.06 & 4.89 & 60.35 & -2.27 & 9.6 & 9.4 & 5.8 & 0.0103 \\
\hline 18 & 515 & 1615 & $"$ & -0.4 & 0.04 & 6.16 & 74.38 & -2.88 & 22.0 & 18 & 8.2 & 0.0049 \\
\hline 19 & 418 & 1400 & $"$ & -0.3 & 0.04 & 6.46 & 101.33 & -2.49 & 30.0 & 12.8 & 9.5 & 0.00422 \\
\hline 20 & 412 & 760 & " & -1.3 & 0.02 & 8.99 & 110.65 & -19.89 & 123.0 & 13.6 & 12.8 & 0.00156 \\
\hline 21 & 511 & 1360 & $"$ & -0.1 & 0.04 & 6.72 & 156.51 & -0.86 & 39.0 & 13.8 & 10.7 & 0.00374 \\
\hline 22 & 492 & 2775 & 0.01099 & -0.3 & 0.05 & 4.70 & 48.39 & -1.26 & 6.6 & 6.3 & 4.1 & 0.0122 \\
\hline 23 & 540 & 2560 & 0.01786 & -0.6 & 0.08 & 4.88 & 48.26 & -2.73 & 12.6 & 7.9 & 5.9 & 0.0136 \\
\hline 24 & 643 & 3050 & 0.0196 & -0.3 & 0.08 & 4.46 & 38.69 & -1.14 & 8.3 & 5.6 & 4.4 & 0.0182 \\
\hline 25 & 565 & 1480 & $"$ & -0.2 & 0.14 & 6.41 & 127.31 & -1.57 & 100 & 22 & 19.6 & 0.00715 \\
\hline 26 & 473 & 855 & 0.0385 & -1.2 & 0.05 & 8.36 & 74.91 & -16.32 & 245 & 7.7 & 7.7 & 0.0065 \\
\hline 27 & $475-538$ & 948 & 0.0399 & -0.732 & 0.07 & 7.83 & 73.4 & -8.8 & 15 & 8 & 8 & 0.0083 \\
\hline 28 & 630 & 2700 & 0.04 & -0.5 & 0.15 & 4.70 & 40.90 & -2.15 & 19.8 & 6.9 & 6.2 & 0.0243 \\
\hline 29 & 495 & 1600 & 0.0625 & -0.3 & 0.10 & 6.03 & 70.34 & -2.18 & 49 & 4.7 & 4.7 & 0.0215 \\
\hline 30 & 755 & 3135 & 0.091 & -0.5 & 0.30 & 4.25 & 24.77 & -1.86 & 25 & 5.7 & 5.8 & 0.0521 \\
\hline 31 & 684 & 2650 & ** & -0.5 & 0.20 & 4.62 & 32.71 & -2.19 & 24.4 & 4.3 & 4.4 & 0.0458 \\
\hline 32 & 653 & 1520 & $"$ & -0.5 & 0.16 & 6.11 & 35.75 & -3.83 & 85 & 5.5 & 5.5 & 0.0290 \\
\hline 33 & 501 & 855 & $"$ & -1.3 & 0.09 & 8.14 & 62,42 & -17.69 & 355 & 5.7 & 5.7 & 0.0157 \\
\hline 34 & 710 & 1635 & $"$ & -0.3 & 0.14 & 5.89 & 81.57 & -2.13 & 60 & 4.5 & 4.5 & 0.0309 \\
\hline 35 & 763 & 1760 & 0.125 & -0.4 & 0.18 & 5.57 & 68.00 & -2.64 & 56 & 3.9 & 4.0 & 0.0446 \\
\hline 36 & 770 & 2700 & 0.167 & -0.5 & 0.28 & 4.40 & 30.76 & -2.15 & 27.5 & 3.3 & 3.5 & 0.0793 \\
\hline 37 & 842 & 1995 & "' & -0.4 & 0.24 & 5.12 & 52.91 & -2.33 & 48 & 3.7 & 3.7 & 0.0644 \\
\hline 38 & 842 & 3135 & 0.2 & -0.5 & 0.36 & 4.00 & 20.68 & -1.86 & 24 & 3.3 & 3.5 & 0.103 \\
\hline 39 & 522 & 855 & $"$ & -1.2 & 0.10 & 7.66 & 48.02 & -16.32 & 246 & 2.9 & 2.9 & 0.0346 \\
\hline 40 & 603 & 1770 & 0.30 & -0.4 & 0.23 & 5.01 & 47.08 & -2.63 & 41 & 2.1 & 2.2 & 0.105 \\
\hline 41 & 650 & 1760 & 0.385 & -0.5 & 0.27 & 4.74 & 43.12 & -3.3 & 37 & 2 & 2 & 0.132 \\
\hline 42 & 671 & 1800 & 0.4 & -0.5 & 0.29 & 4.63 & 41.36 & -3.23 & 36 & 2 & 2 & 0.140 \\
\hline 43 & $558-600$ & 991 & 0.425 & -0.755 & 0.09 & 5.93 & 58. & -8.7 & 2 & 1 & 1 & 0.0934 \\
\hline 44 & 980 & 2800 & 0.5 & -0.5 & 0.41 & 3.43 & 22.35 & -2.08 & 15.5 & 1.7 & 1.8 & 0.229 \\
\hline 45 & 764 & 2000 & $"$ & -0.5 & 0.31 & 4.06 & 34.49 & -2.91 & 22 & 1.6 & 1.6 & 0.187 \\
\hline 46 & 592 & 840 & $"$ & -1.2 & 0.14 & 6.26 & 37.25 & -16.62 & 86 & 1.7 & 1.7 & 0.0838 \\
\hline 47 & 1028 & 2800 & 0.588 & -0.5 & 0.43 & 3.15 & 22.43 & -2.08 & 12.3 & 1.5 & 1.6 & 0.268 \\
\hline 48 & 759 & 1540 & 0.625 & -0.5 & 0.40 & 4.08 & 30.45 & -3.78 & 29 & 2 & 2 & 0.193 \\
\hline 49 & 611 & 810 & 0.667 & -1.2 & 0.17 & 5.36 & 35.82 & -17.23 & 43 & 1.6 & 1.6 & 0.106 \\
\hline 50 & 822 & 1500 & 0.833 & -0.5 & 0.40 & 3.00 & 30.53 & $-3,88$ & 9 & 1.6 & 1.6 & 0.252 \\
\hline 51 & 888 & 1320 & 0.909 & -0.4 & 0.40 & 2.63 & 30.81 & -3.52 & 6.8 & 1.6 & 1.6 & 0.247 \\
\hline 52 & 705 & 760 & & -1.2 & 0.19 & 3.66 & 35.93 & -18.36 & 9 & 1.6 & 1.6 & 0.115 \\
\hline
\end{tabular}

current $\left(I_{+}\right)$measured in the range $155^{\circ} \leq \theta<180^{\circ}$ (Brace et al., 1973; SAMIR et al., 1979a). The average ionic Mach number $\left(S_{\mathrm{AVV}}\right)$ was obtained from

$$
S_{\mathrm{AV}}=\frac{V_{s}}{\sqrt{\left[\frac{2 \mathrm{k} T_{e}}{M_{(\mathrm{av})}}\right]}}=\frac{\left\{V_{\mathrm{s}}\left[\frac{(16 R+1)}{(R+1)}\right]^{1 / 2}\right\}}{\left(2 \mathrm{k} T_{e}\right)^{1 / 2}}
$$

where: $R=\frac{\left[N\left(\mathrm{O}^{+}\right)\right]}{\left[N\left(\mathrm{H}^{+}\right)\right]}$. In the present study we assume that $\beta=\frac{T_{e}}{T_{+}}=1$. The present investigation covers the plasma property ranges:

$$
\begin{aligned}
1 \times 10^{4} & \leq\left[N_{+}(\text {ambient })\right] \leq 6.8 \times 10^{5} \mathrm{~cm}^{-3} \\
760^{\circ} \mathrm{K} & \leq\left[T_{e}(\text { ambient })\right] \leq 3135^{\circ} \mathrm{K} \\
0.1 & \leq\left|\phi_{s}\right| \leq 1.3 \mathrm{~V},
\end{aligned}
$$


and the plasma parameter ranges:

$$
\begin{gathered}
31 \leq R_{\mathrm{D}} \leq 216 \\
2.6 \leq S_{\mathrm{AV}} \leq 9.0 \\
0.85 \leq\left|\phi_{\mathrm{N}}\right| \leq 19.9 \\
1 \times 10^{-1} \leq R \leq 10^{3} .
\end{gathered}
$$

\subsection{Theoretical models}

In order to compute the electron density, ion density and the potential at any point in the flow field around a rapidly moving conductor in a rarefied plasma, the Vlasov-Poisson system of equations (written for each component of the plasma) should be solved in a self-consistent manner. Since this is a difficult mathematical task simplifying physical assumptions and mathematical approximations are called upon. Often, such simplifications make the solution for the case of a satellite moving in the terrestrial ionosphere unrealistic.

In Gurevich et al., 1970, the flow of rarefied plasmas over bodies of different shapes is computed. A comparison of such models with some experimental results from the Ariel 1 (SAMIR and WILLMORE, 1965; HENDERSON and SAMIR, 1967) and from the Explorer 31 satellite (SAMIR and WRENN 1969), are also given. The theoretical models, including their validity ranges, the physical assumptions and the mathematical approximations are discussed in detail in AL'PERT, 1976. Results of an attempt to compare an elaborate numerical wake model (PARKER, 1976) with some of the above experimental results are given by PARKeR (1977). Unfortunately, the latter has not yet been shown to be practically superior to the much simpler models given by GuREvich et al, 1970 and AL'PERT, 1976 and used in theory-experiment comparisons with some Ariel 1 and Explorer 31 experimental results. It is therefore very tempting to use the sample of data given in Table 1 (after: SAMIR et al., 1979a, b) and attempt a comparison with some models given in Gurevich et al., (1970).

Since we are interested in a theory-experiment comparison using the measurements from the C.E.P. probe on the AE-C satellite, we have modified the treatment of Gurevich et al., (1970), AL'PERT (1976) (given for a very long circular cylinder with $R_{0} \gg \lambda_{\mathrm{D}}$ ), so as to 'accommodate' for the location of the probe on the satellite.

While we are fully aware of the limitation of such a comparison, we are interested to find whether simple theories, composed essentially of analytical equations can predict the quantitative trend of the

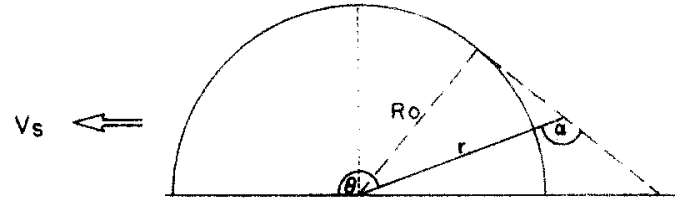

Fig. 1. Angular and Radii rotation used.

experimental results and assess (on a relative basis) which of the plasma properties or parameters have the major influence on the distribution of ions in the wake of an ionospheric satellite. This approach, despite its limitation, may be sufficiently beneficial and adequate for practical cases in future spacelab missions. The theoretical expressions used here are based on: (1) the quasi-neutral approximation given in the Appendix as equation (A-1), and (2) on equation (A-2), for the neutral approximation.

The quasi-neutral $(q)$ approximation can he written in the form.

$$
\begin{aligned}
n^{\mathrm{a}}(\theta)^{*}= & 0.71\left\{\exp \left[-\sqrt{2} S(\theta-\alpha)-1 / 2(\theta-\alpha)^{2}\right]\right. \\
& \left.+k \exp \left[-S(\theta+\alpha)-1 / 2(\theta+\alpha)^{2}\right]\right\},
\end{aligned}
$$

where $\mathrm{k}=1$ for $\theta-\alpha \leq \pi / 2, \mathrm{k}=0$ for $\theta-\alpha>\pi / 2, \alpha$ is the shift angle appropriate to the distance of the collection area of the probe from the body (Fig. 1) and is given by:

$$
\alpha=\pi-\sin ^{-1}\left(R_{0} / r\right)
$$

Therefore $\alpha$ relates the density of ions at a distance $r$ from the body with the density at the body's surface. We prefer to find $\alpha$ from the experimental results given by SAMIR et al. (1979b). The $\alpha$ is the angle at which the ion current begins to meaningfully deviate from its ambient value (SAMIR et al., 1979b).

For a mixture of hydrogen and oxygen ions each one with a different value of the Mach number $S$, the combined ion concentration is given as a weighted sum of the contribution of each component:

$$
n^{\mathrm{q}}(\theta)=W_{\mathrm{O}^{+}} n_{\mathrm{O}^{+}}(\theta)+W_{\mathrm{H}^{+}} n_{\mathrm{H}^{+}}{ }^{a}(\theta) .
$$

where $W_{\mathrm{O}^{+}}$and $W_{\mathrm{H}^{+}}$are the relative concentrations of oxygen and hydrogen ions respectively $(=$ nondimensional quantity). The neutral approximation is written in the form:

$$
n^{n}(\theta)=\left[1+\Phi\left(S \cos \phi_{0} \cos \theta^{\prime}\right)\right] /\left[1+\Phi\left(S \cos \phi_{0}\right)\right] \text {, }
$$

\footnotetext{
*Note: $n$ is a normalized (nondimensional) quantity. Thus $n^{a}(\theta)=\left\{\frac{[N(\theta)]}{N_{0}}\right\}^{q} \equiv$ normalized density computed according to the quasineutral approximation.
} 
where

$$
\begin{array}{r}
\cos \phi_{0} \approx \frac{1}{\sqrt{2}}, \quad \Phi(x)=\frac{2}{\sqrt{\pi}} \int_{0}^{x} e^{-\left(x^{\prime}\right)^{x} \mathrm{~d} x^{\prime}} \\
(=\text { the 'error function') }
\end{array}
$$

the value of $\theta^{r}$ is taken by:

$$
\theta^{\prime}=\theta-\left(\alpha-\frac{\pi}{2}\right)
$$

in order to take into account the shift due to the distance of the probe from the surface body. The shift in the quasi-neutral approximation was taken as $\alpha$ while in the neutral approximation as $\alpha-\frac{\pi}{2}$, because the ambient value is obtained in these approximations at the angle of attack 0 and $\frac{\pi}{2}$ respectively. The contribution of hydrogen ions and oxygen ions is taken into account in a similar way to equation (3):

$$
n^{n}(\theta)=W_{\mathrm{O}^{+}} n_{\mathrm{O}^{+}}{ }^{n}(\theta)+W_{\mathrm{H}^{+}} n_{\mathrm{H}^{+}}{ }^{n}(\theta)
$$

The comparison of the measured current ratios and the theoretical density ratios is done under the approximation:

$$
I(\theta) / I(\alpha)=[n(\theta)] /[n(\alpha)],
$$

where the ambient value was taken at $\theta=\alpha$ which is the highest value of the angle of attack where the current is closest to the ambient current (SAMIR et al., 1979a). The approximation (7) can be questioned and indeed it imposes a limitation on our study. This matter was discussed in detail by SAMIR et al., (1979b).

From the assumptions on which the quasi-neutral approximation and the neutral approximation are based (Gurevich et al., 1970), it follows that the quasi-neutral approximation has to be best suitable for oxygen ions while the neutral approximation has to be best suitable for hydrogen ions. Therefore we shall compare the experimental results with the 'combined approximation':

$$
n^{c}(\theta)=W_{\mathrm{O}^{+}} \frac{\left[n_{\mathrm{O}^{+}}{ }^{q}(\theta)\right]}{\left[n_{\mathrm{O}^{+}}{ }^{a}(\alpha)\right]}+W_{\mathrm{H}^{+}} \frac{\left[n_{\mathrm{H}^{+}}{ }^{n}(\theta)\right]}{\left[n_{\mathrm{H}^{+}}{ }^{n}(\alpha)\right]},
$$

which considers the contribution of oxygen ions by the quasi-neutral approximation, and the contribution of the hydrogen ions by the neutral approximation.

\section{SOME THEORY-EXXERMMENT COMPARISONS}

As mentioned earlier, the experimental results used here consist of those given in SAMIR et al, 1979a and 1979b. In SAmr et al., 1979b six cases were used where the ion current was determined as a function of the angle of attack $(\theta)$, in the angular range: $90^{\circ} \leq \theta \leq 160^{\circ}$. It was shown there that the 'ambient' current at $\theta=90^{\circ}$ is not very different from the current at $\theta \sim 120^{\circ}$. The value of $\alpha$ was therefore taken at $\theta=120^{\circ}$.

Figure 2 shows the variation of the normalized ion current with angle of attack $(\theta)$ for $90^{\circ} \leq \theta \leq$ $160^{\circ}$ which (as explained above) is in fact for $120^{\circ} \leq \theta \leq 160^{\circ}$. We will focus here on the comparison of the quasi-neutral and neutral approximations with the experimental results in terms of the concentration of hydrogen. The experimental results of: $[I(\theta)] /[I(\alpha)]$ are given by crosses when the angle of attack was changed from $90^{\circ}$ to $160^{\circ}$ and by full circles when the angle of attack was changed from $160^{\circ}$ to $90^{\circ}$. The results of the neutral, quasineutral and combined approximations are shown by the unequally dashed, equally dashed and solid lines respectively. In Fig. $2 a, 2 c$ and $2 e$ where a small amount of hydrogen is present, (of the order of $10^{-3}$ ) it is seen that the quasi-neutral approximation is closer to the experimental values. On the other hand, the slopes of the neutral approximation (in all but Fig. 2a) show trends more similar to those shown by the experimental results (compared with the trend due to the quasi-neutral approximation). The combined approximation gives the best results for all the six cases.

The general characteristic of the theoretical models (Fig. 2) is that they give values lower than the observed. As seen, the difference between the theoretical and the experimental results increase with decreasing percentage of $\left[\mathrm{H}^{+}\right]$.

Detailed values for the theory-experiment comparison are given in Table 1. In this table, the experimental results are ordered with increasing values of $\left(W_{\mathrm{H}^{+}}\right)$. The results consist of the six cases given in SAMIR et al., 1979b (and these are presented in terms of altitude ranges in the first column on the left) and the 46 cases after SAMr et al., $1979 \mathrm{a}$. For the latter, the latitude refers to the exact height of the measurement. Cases with similar $\left[\mathrm{H}^{+}\right]$concentration are ordered according to increasing values of $R_{\mathrm{D}}$.

The first group (cases 1 to 13 ) in Table 1 presents cases for which $W_{\mathrm{H}^{+}}<2 \times 10^{-3}$. It is seen that the ratio between the experimental results to the theoretical ones $(\rho)$ is smallest for the combined approximation $\left(\rho^{c}\right)$ with $\rho^{q}<\rho^{n}$ (where: $\rho^{q}=$ ratio for quasi-neutral approximation and $\rho^{n}=$ ratio for neutral approximation).

The second group (cases 14-25) for which $2 \times$ $10^{-3}<W_{H^{+}}<2 \times 10^{-2}$, shows the $\rho^{c}$ values to be the smallest, i.e. the combined approximation is 
(a)

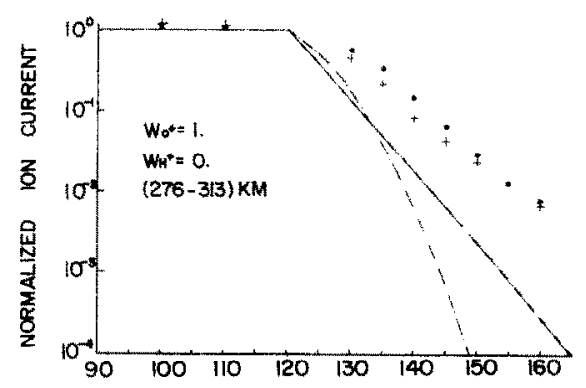

(b)

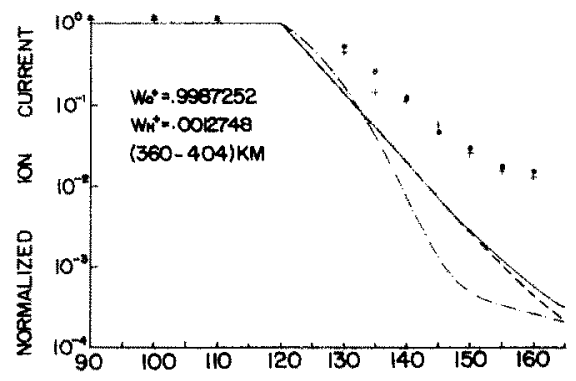

(c)

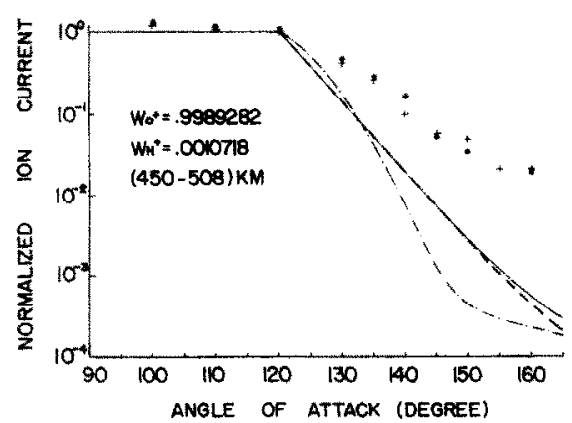

(d)

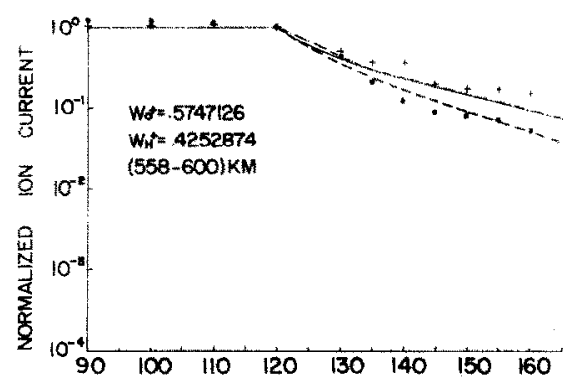

(e)

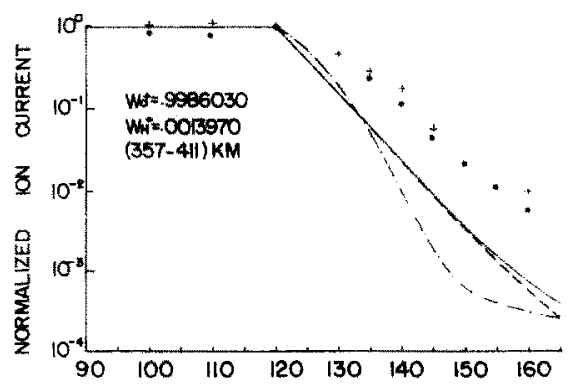

(f)

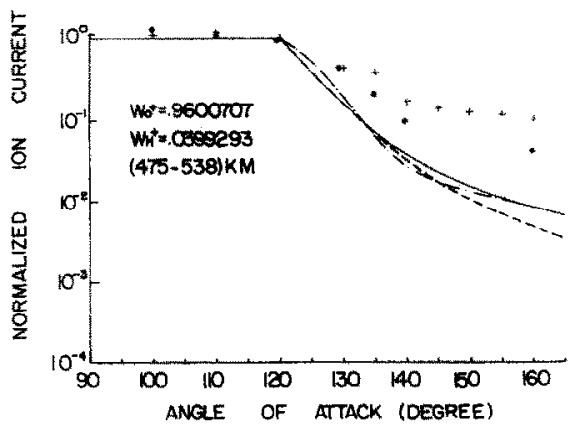

Fig. 2. The variation of "Normalized ion current" $\left[=\frac{i(\theta)}{i\left(120^{\circ}\right)}\right]$ with angle of attack $(\theta)$ in the angular range $90^{\circ} \leq \theta \leq 160^{\circ}$. The $(+)$ data represents the situation when the probe rotated from $90^{\circ}$ to $160^{\circ}$ and the (O) data represent the situation when the probe rotated from $160^{\circ}$ to $90^{\circ}$. The semi-dashed line represents the neutral approximation and the solid line represents the combined approximation. The quasi-neutral approximation is given by a dashed line. Hydrogen and oxygen concentrations and altitude ranges are given in the inserts. Other parameters are tabulated in Table 1 . Note: this figure provides both the experimental and the theoretical information for the six cases given in SAMIR et al.

(1979(b)), in the altitude range $276-600 \mathrm{~km}$.

closest to the experimental results. For the third group (cases 26-52) where: $3 \times 10^{2}<W_{\mathbf{H}^{+}} \leq$ $9 \times 10^{-1}$ we find: $\rho^{c}-\rho^{n}$ with $\rho^{c}$ and $\rho^{n}$ approaching unity as $W_{H^{*}}$ increases towards unity.

If we are to accept (at the present time), $\rho<10$ as an indicator for "rough applicability" of a theoretical model to in situ experimental results, then we find: $\rho<10$ for $W_{\mathrm{H}^{*}}>3 \times 10^{-2}$ and for any value of the other parameters and $\rho^{c} \approx \rho^{n}$ for the above condition of $W_{\mathrm{H}}$. Moreover, we find: $\rho^{c}<$ 10 for $T_{e}>1500^{\circ} \mathrm{K}$. It appears therefore that the applicability of the combined approximation model to measurements made at a distance of about $\left(\frac{R_{0}}{2}\right)$ 


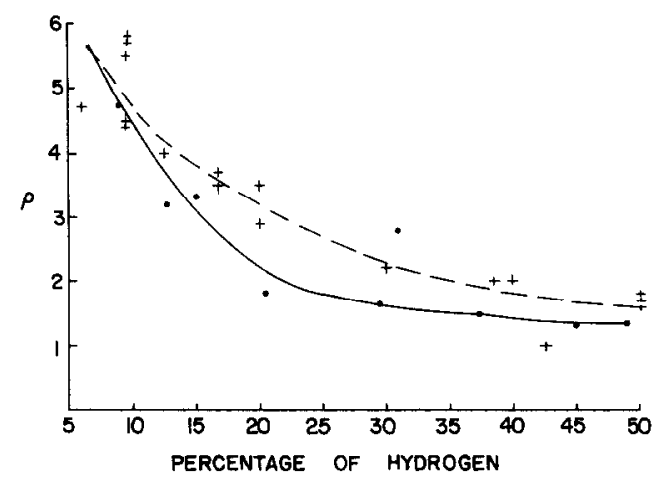

Fig. 3. The variation of the ratio between the experimental results $\left(\gamma_{\text {EXP }}\right)$ and the theoretical approximation $\left(\rho^{c}\right)$ with hydrogen concentration $\left(W_{\mathbf{H}^{+}}\right)$. The crosses $(+)$refer to the present study and the full circles to the results of SAMIR et al. (1975).

from the surface of the satellite is essentially similar to the degree of applicability of the neutral approximation to results obtained by a (surface) flushmounted probe (SAMIR et al., 1975). The main difference in the theoretical approximation used in the present study to that used by SAMIR et al. (1975) is in the angle $\alpha$ which was not used in the latter paper since the probe was flush-mounted on the surface of the Explorer 31 satellite. The behavior of $I_{+}=f\left(W_{\mathrm{H}^{+}}\right)$in the present study is in accord with that shown in SAMIR et al. (1975), and with AL'PERT (1976). Figure 3 shows the degree of agreement between theory and experiment based on the large sample of results used in the present study with that used in SAMIR et al. (1975). It is seen that the behavior of the ratio $(\rho)$ is similar in both studies. For example, this ratio decreases from $\rho=5$ for $W_{\mathrm{H}^{+}}=0.05(\approx 5 \%)$ down to $\rho=1.5$ for $W_{\mathbf{H}^{+}}=0.5(=50 \%)$. The similar behavior for the surface mounted probe on the Explorer 31 satellite (SAmIR et al., 1975) and the cylindrical probe (BRACE et al., 1973; SAMIr et al., 1979a and 1979b) on the AE-C satellite indicate that the angle $(\alpha)$ represents correctly the influence of probe location and distance (w.r.t. the satellite surface) on the ion currents in the wake.

As seen from Table 1, the combined approximation $\left(\rho^{c}\right)$ behaves in a similar way to the quasineutral approximation in the first region and to the neutral approximation in the third region in providing the 'closest' agreement with the experimental results.

We now examine the relative importance of $\phi_{\mathrm{N}}$ on the variation of $\rho^{c}$ with $W_{\mathbf{H}^{+}}$.

From Fig. 4 it is seen that in region III the spread of $\rho^{c}\left[=f\left(W_{\mathbf{H}^{+}}\right)\right]$is small compared with the spread of $\rho^{c}$ in regions II and I. This implies that for region III $\rho^{c}$ depends solely on $W_{\mathbf{H}^{+}}$and the other parameters are of less significance. On the other hand in region $I$, the $\rho^{c}$ depends on other parameters.

Now we examine whether it is more meaningful to express the ratio $\gamma_{\mathrm{EXP}}$ in terms of plasma properties (e.g. $T_{e}, \phi_{s}, W_{\mathrm{H}^{+}}$) or parameters (e.g. $R_{\mathrm{D}}, \phi_{\mathrm{N}}$, $S_{\mathrm{AV}}$ ). It is clear from Table 1 and Fig. 4 that the plasma property that influences most the experimental results is $W_{\mathrm{H}^{+}}$. Figure 5 shows the variation of $\gamma_{E X P}$ with $W_{\mathrm{H}^{+}}$. It is seen that for $W_{\mathrm{H}^{+}} \leq 0.01$, the $\gamma_{\mathrm{EXP}}$ are very small.

In this range there is no clear dependence of the

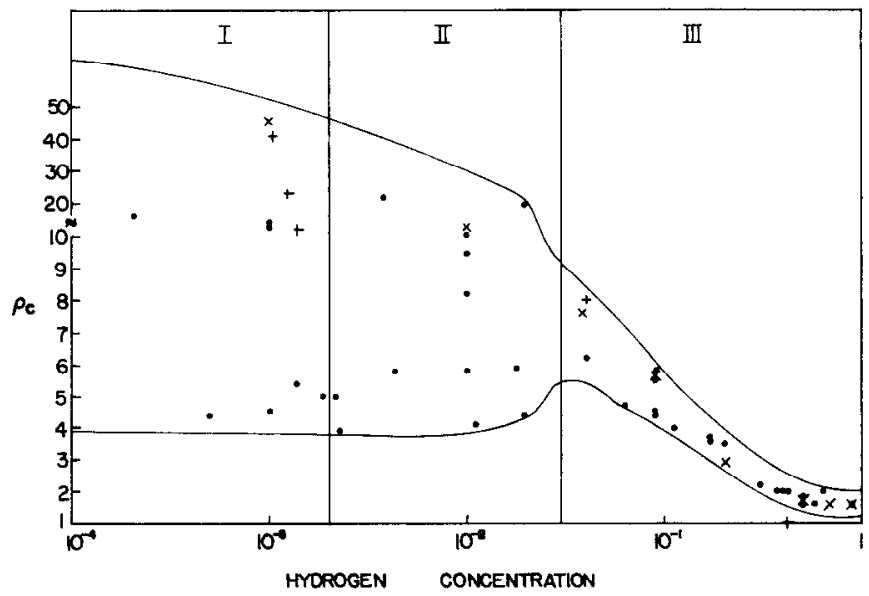

Fig. 4. The variation of the ratio between the experimental results and the combined approximation $\left(\rho^{c}\right)$ with $W_{\mathrm{H}^{+}}$. Full circles show results for $\phi_{\mathrm{N}}<3$, crosses show results for $\phi_{N} \sim 9$ and $x$ notation stands for $\phi_{N} \sim 16$. The solid lines show the envelope of the results in the $\left(\rho^{c}, W_{\mathbf{H}^{+}}\right)$plane. 


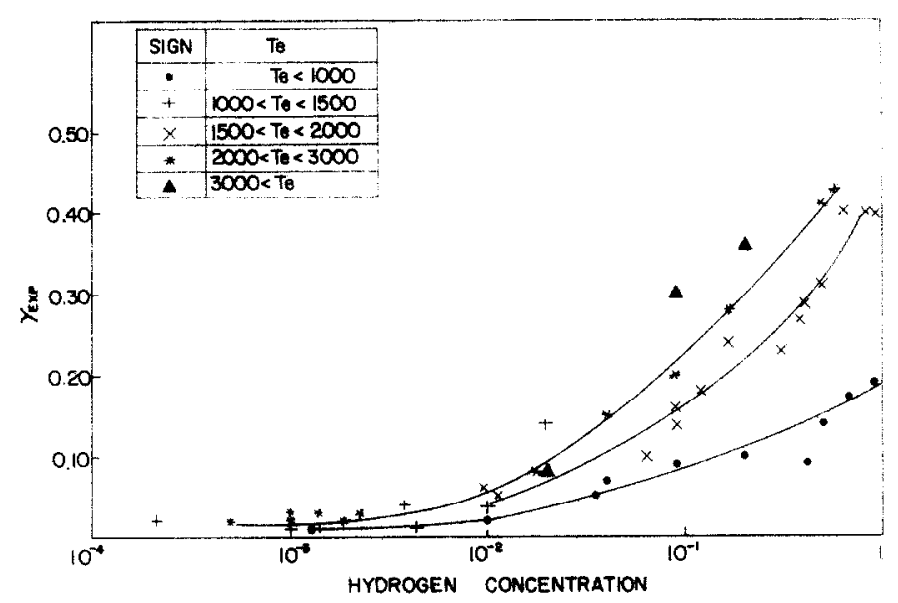

Fig. 5. The variation of the experimental results $\left(\gamma_{\mathrm{EXP}}\right)$ with $W_{\mathrm{H}^{+}}$for five ranges of temperatures. The solid lines depict the slopes.

experimental results on the hydrogen concentration of electron temperature, thus it follows that in this region, the oxygen ions determine the wake current. This is of great importance at low altitudes.

For $W_{\mathbf{H}^{+}}>0.01, \gamma_{\mathrm{Exp}}$ increases and the slope of $\gamma_{\mathrm{EXP}}=f\left(W_{\mathrm{H}^{+}}\right)$is $T_{e}$ dependent, such that for higher values of $T_{e}$ the slope is steeper.

Also, $T_{e}$ appears more significant compared with $\phi_{s}$ at a distance of $\left(R_{0} / 2\right)$ from the surface of the satellite. This follows from the grouping of the $\gamma_{\text {EXP }}$ values along the solid lines which shows little fluctuations. The only exception may be the $\gamma_{E X P}$ for $W_{\mathrm{H}^{+}}=0.02$ which corresponds to cases No. 24 and No. 25. For these cases there is nothing in the values of the other parameters, that can indicate an influence of a different parameter tabulated in Table 1.

From the above discussion follows that the most convenient way to explain the behavior of the present experimental results is in terms of $\mathrm{W}_{\mathrm{H}^{+}}$and $T_{e}$. For the region where $W_{\mathrm{H}^{+}}<2 \times 10^{-3}$ we examined $\gamma_{\mathrm{EXP}}=f\left(\mathrm{~W}_{\mathrm{H}^{+}}, T_{e}, \phi_{s}, \phi_{\mathrm{N}}, R_{\mathrm{D}}, S_{\mathrm{AV}}\right)$ and found, that $\gamma_{E X P}=f\left(T_{e}\right)$ was the prominent variation (Figs. 6 and 7). In fact $\gamma_{\mathrm{EXP}}=f\left(T_{e}\right)$ is linear. The encircled points in Figs. 6 and 7 refer to high values of plasma potential. For $\gamma_{\mathrm{EXP}}=f\left(T_{e}\right)$ they increase $\gamma_{E X P}$ by about a factor of 2 . This is reasonable since for lower particle-temperature (i.e. lower energy) the significance of the plasma potential increases.

A similar examination was performed for plasmas with $W_{\mathbf{H}^{+}}=1 \times 10^{-2}$ and $W_{\mathbf{H}^{+}}=9 \times 10^{-2}$. This is shown in Figs. 8 and 9. From Fig. 8 we see that $\gamma_{E X P}$ is dependent on $T_{e}$ for both plasmas, similar to the relationship seen for plasmas with $W_{\mathrm{H}^{+}}<$
$2 \times 10^{-3}$ (Fig. 6). Also, it appears that there is a dependence on the spacecraft potential $\left(\phi_{s}\right)$ for $\left|\phi_{s}\right| \leq 0.5$. The situation for $\gamma_{\mathrm{EXP}}=f\left(\left|\phi_{s}\right|\right)$ for $\left|\phi_{\mathrm{s}}\right|>$ 0.5 is not clear. From Fig. 9 we see that $\gamma_{\text {EXP }}$ decreases with increasing $R_{\mathrm{D}}$ and $S_{\mathrm{AV}}$. Hence, the

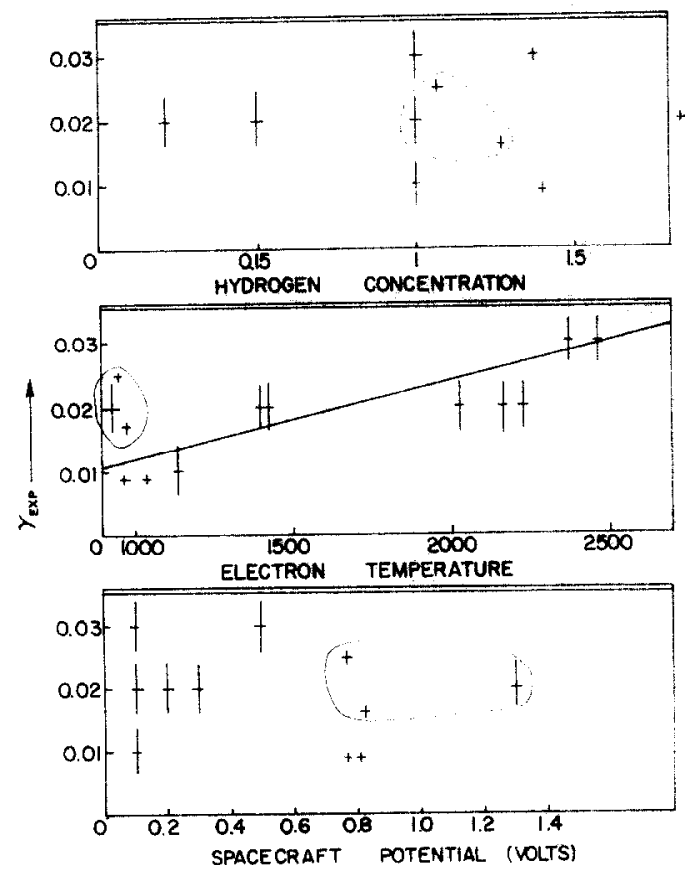

Fig. 6. The dependence of $\gamma_{\text {EXP }}$ on some plasma properties (hydrogen concentration and electron temperature) and body potential. The encircled points show the position of three experimental values of different behavior discussed in the text. Results are given for hydrogen concentration less than $2 \times 10^{-3}$. 


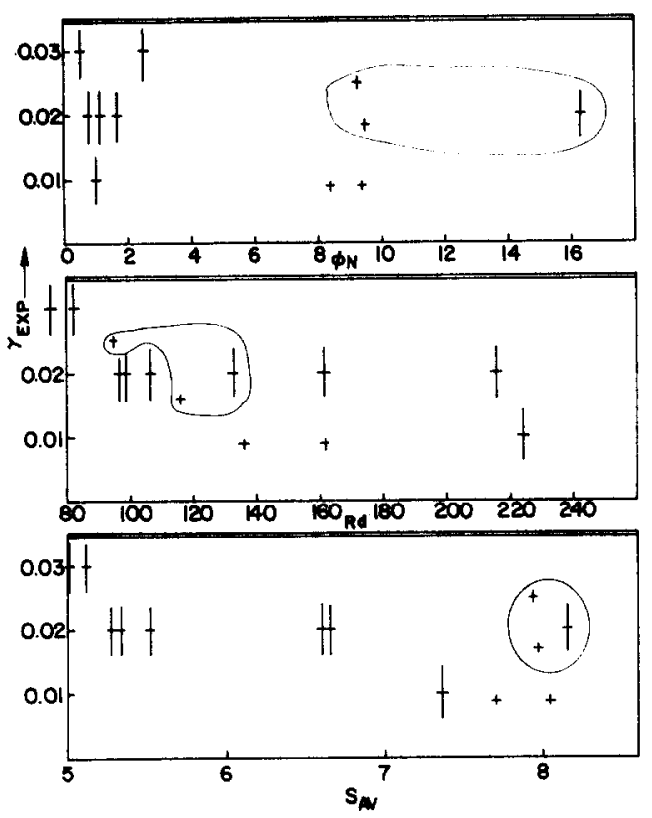

Fig. 7. The dependence of $\gamma_{\text {ExP }}$ on the plasma parameters $\left(\phi_{N}, R_{\mathrm{D}}\right.$ and $\left.S_{\mathrm{AV}}\right)$ for $W_{\mathrm{H}^{+}}<2 \times 10^{-3}$. The encircled results are the same as in Fig. 5 .

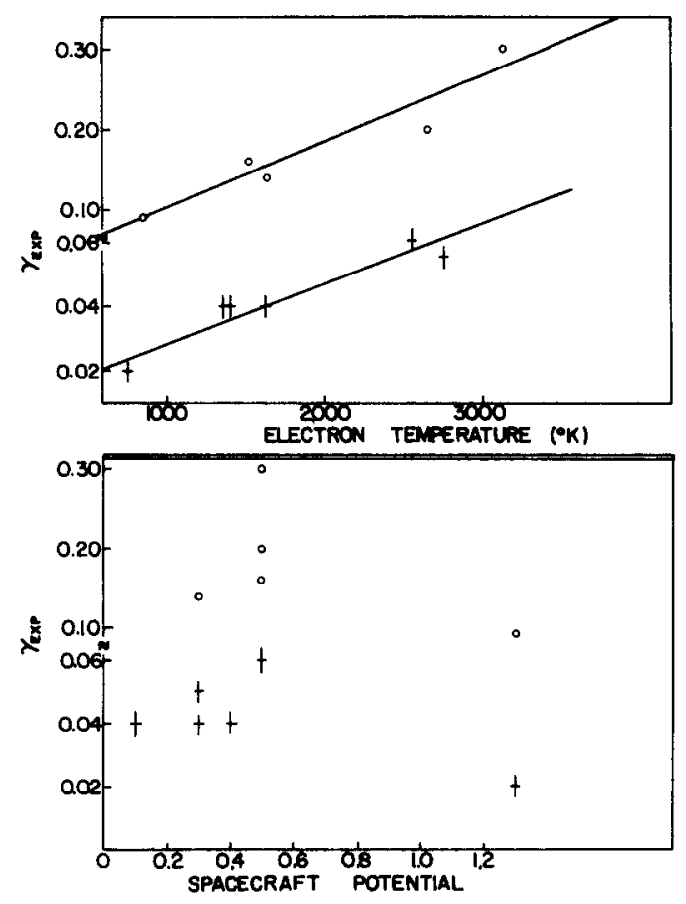

Fig. 8. Same as Fig. 5 but for two hydrogen concentrations $W_{\mathbf{H}^{+}}=1 \times 10^{-2}$ (crosses) and $W_{\mathbf{H}^{+}}=9 \times 10^{-2}$ (circles).
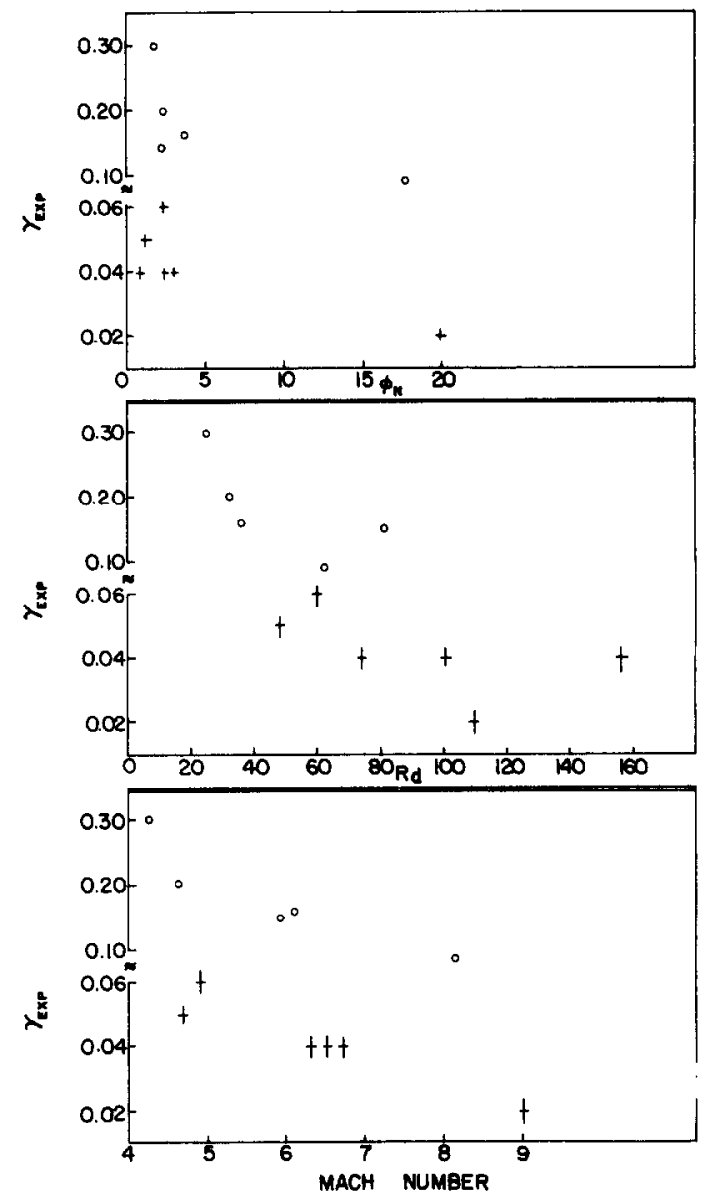

Fig. 9. Same as Fig. 6 but for two hydrogen concentrations $W_{\mathbf{H}^{+}}=1 \times 10^{-2}$ (crosses) and $9 \times 10^{-2}$ (circles).

behavior of $\gamma_{\mathrm{EXP}}$ for these plasmas should be given in terms of plasma flow parameters and at the present time it is difficult to give a unique answer as to which of the parameters is more significant. Figure 10 shows the variation of $\gamma_{N}\left(\equiv \gamma / W_{\mathrm{H}^{+}}\right)$with electron temperature, for $W_{\mathrm{H}^{+}}$in the range $0.5 \leq$ $W_{\mathrm{H}^{+}}<1.0$. Both the solid and the dashed curves are results of computation while the crosses $(+)$ depict the experimental data.

Since the $W_{\mathrm{H}^{+}}$is different from case to case it was assumed that $\gamma_{\mathrm{EXP}}$ is proportional to the relative hydrogen concentration. This assumption is justified since the ambient current is proportional to the total ion concentration, while the ion current in the wake is proportional mainly to the hydrogen concentration. For $W_{\mathrm{H}^{+}} \geq 50 \%$ the contribution of oxygen ions to the current in the wake is negligible. Therefore $\gamma_{\mathrm{EXP}}$ is proportional to $W_{\mathbf{H}^{+}}$.

It follows from the previous discussion that $\gamma_{\mathrm{EXP}}^{N}$ corresponds to $\gamma_{\mathrm{EXP}}$ when $W_{\mathrm{H}^{+}}=1.0$. The solid line 


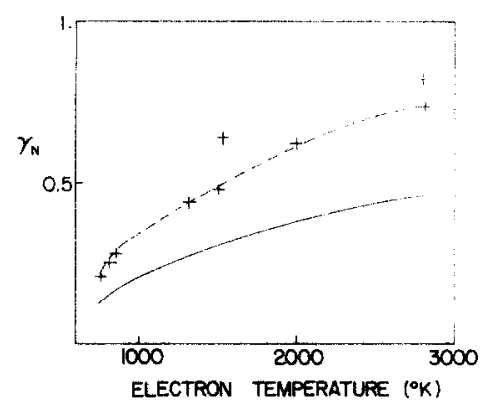

Fig. 10. The variation of $\gamma_{N}\left(\equiv \gamma / W_{H^{+}}\right)$with electron temperature for $0.5 \leq W_{\mathrm{H}^{+}}<1.0$. The solid line depicts the computation due to the combined approximation and the dashed line is the latter multiplied by the factor 1.6.

in Fig. 10 shows the $\rho$ approximation. It appears that a multiplication of the latter curve by 1.6 provide excellent agreement with $\gamma_{\text {EXP. }}^{N}$. Hence the difference between the theoretical computation and $\gamma_{\mathrm{EXP}}^{N}$ is by a constant $=1.6$. The three points on the lower left side of this figure correspond to high potentials $\left(\phi_{N} \approx 16-18\right)$ whereas the other points refer to lower potentials $\left(\phi_{N} \approx 2-4\right)$.

\section{SUMMARY}

Experimental results of 52 cases were used in order to assess the degree and range of applicability of some simple wake models in the study of satellite-ionosphere interactions. The theoretical models used are extensions of early models, and are based on a quasi-neutral approximation for the oxygen ions and a neutral approximation for the hydrogen ions, taking into account the distance between the probe and the body by an appropriate shift angle.

For ionospheric plasmas with hydrogen concentrations greater than $3 \%$ or alternatively for electron temperature larger than $1500^{\circ} \mathrm{K}$, it is found that the ratio between the experimental results $\left(\gamma_{E X P}\right)$ and the theoretical approximation $\left(\rho^{c}\right)$ is less than a factor of 10 .

Note that for the purpose of this study we consider a difference of 1.6 between theory and experiment to imply very good agreement.

The trend of $\gamma=f\left(\theta, W_{H^{\prime}}, T_{e}\right)$ for both theory and experiment is similar. The examination of $\gamma_{\mathrm{EXP}}$ variation with plasma properties and flow parameters showed that $W_{\mathrm{H}^{+}}$and $T_{e}$ are most significant. The spacecraft potential had only a slight influence at small hydrogen concentrations and small electron temperature. The examination of the realistic influence of the flow parameters does not provide unique answers as to the relative significance of all the parameters. It appears that the variations of $\gamma_{E X P}$ can be best described in terms of $W_{14}$ and $T$. However, further systematic investigations are $\mathrm{rc}$. quired in order to solidly substantiate the above.

It is expected that theory-experiment assessments such as performed here should be useful tor the planning of plasma flow interaction experiments in the Shuttle-Spacelab era as well as to the better understanding of body-plasma interactions in a supersonic and sub-Alfvenic flow regime

Acknowledgements-One of the authors (U. S.) acknowledges the support of NSF grant ATM $76-19790$ and NASA grant NGR 23-005-320. The pleasant collaboration with L. BRACE and H. Brinton from G.S.F.C in utilizing the relevant measurements from the AE-C satellite is highly appreciated.

\section{APPENDIX}

\section{Expression A-1}

In the present investigation we have chosen to compare some of our experimental results from the AE-C satellite (SAmIR et al., 1979) as given in Table 1 with two fairly simple theoretical wake models given by:

$$
\begin{aligned}
n^{\mathrm{q}}(\theta)= & \left\{\frac{[N(r, \theta)]}{N_{0}}\right\} \\
= & C\left(\frac{T_{e}}{T_{+}}\right)\left\{\operatorname { e x p } \left[-\frac{V_{0}}{V_{s}}\left(\pi-\theta-\arcsin \frac{\rho_{0}}{r}\right)\right.\right. \\
& \left.-1 / 2\left(\pi-\theta-\arcsin \frac{\rho_{0}}{r}\right)^{2}\right] \\
& +\mathrm{K} \exp \left[-\frac{V_{0}}{V_{*}}\left(\pi-\theta+\arcsin \frac{\rho_{0}}{r}\right)\right. \\
& \left.\left.-1 / 2\left(\pi-\theta+\arcsin \frac{\rho_{0}}{r}\right)^{2}\right]\right\},
\end{aligned}
$$

where: $c\left(T_{e} / T_{+}\right)=0.7-0.4$ for $\left(T_{e} / T_{+}\right)$varying between one and large values. The constant $K$ is equal to either 1 or 0 according to whether the angle $\theta$ (between the radius vector and the $-V_{0}$ direction is greater or less than $\left(\pi / 2-\arcsin \rho_{0} / r\right)$ i.e. whether the observation point lies outside or inside the geometric shadow of the body (which in this case is a cylinder whose circular radius $\left.\rho_{0} \gg \lambda_{D}\right)$, as formed by the tangents to its cross-section parallel to its velocity vector (AL'PERT, 1976; GUREvich et al., 1970).

Expression (A-1) written for $\theta=0^{\circ}$ (i.e. on the axis) behind a round disc with $\rho_{0}>\lambda_{\mathrm{D}}$ was used by GuREVICH et al. (1970) in a comparison with measured electron currents from the Ariel 1 satellite. The measurements were made by a probe flush-mounted on the surface of the satellite. The theoretical model (A-1) seems to describe the experimental trend $I_{e}=f(\theta)$ quite reasonably but for the maximum rarefaction region, i.e. on the axis behind the satellite) there was a quantitative difference (e.g. AL'PERT, 1976). However, the comparison of (A-1) with the experimental results yielded values which are by 
several orders of magnitude better than those obtained by the conventional 'neutral approximation' (see for example: SAMIR et al., 1975; Al'PERT. 1976). The same expression was used for comparison with some experimental results from the Explorer 31 satellite (SAMIR and WRENN, 1969). For specific cases, in particular when the ionospheric plasma contained mainly $\mathrm{O}^{+}$ions the (A-1) was also very useful and displayed the trend of the experimental results but yielded values different to those obtained in the experiment for the maximum rarefaction region (e.g. AL'PERT, 1976).

\section{Expression A-2}

For ionospheric plasmas with $\mathrm{H}^{+}$as the major ionic constituent, GuREvich et al. used the expression (A-2) given by:

$$
\begin{aligned}
& n^{n}(\theta)=\left\{[N(\theta)] / N_{0}\right\}=N\left(\mathrm{O}^{+}\right) / \\
& \quad N_{0}\left\{\left[1+\operatorname{erf}\left(S_{\mathrm{O}^{+}} \cdot \cos \phi_{0} \cos \theta\right)\right] /\left[1+\operatorname{erf}\left(S_{\mathrm{O}^{+}}+\cos \phi_{0}\right)\right]\right\} \\
& +\left[N\left(\mathrm{H}^{+}\right)\right] / N_{0}\left\{\left[1+\operatorname{erf}\left(S_{\mathrm{H}^{+}}+\cos \phi_{0} \cos \theta\right)\right] /\right. \\
& \left.\left.1+\operatorname{erf}\left(S_{\mathbf{H}^{+}} \cos \phi_{0}\right)\right]\right\} \quad(\mathrm{A}-2)
\end{aligned}
$$

where: $\Phi(z)=e r f(z)=2 / \sqrt{ } \pi \int_{0}^{z} e^{-t^{2}} \mathrm{~d} t$ and $S_{\mathrm{O}^{+}}$and $S_{\mathrm{H}^{+}}$represent the ionic Mach number for $\mathrm{O}^{+}$and $\mathrm{H}^{+}$ions respectively. Expression (A-2) is expected to be valid when the relative value of protons $\left(\mathrm{H}^{+}\right)$in the plasma exceeds $30 \%$ (AL'PERT, 1976). It should be noticed that (A-2) is essentially a 'neutral-approximation' expression. It was shown by SAMIR et al., 1975 that (A-2) provides a reasonable description for electron currents in the wake (this is discussed in greater detail in AL'PERT, 1976).

\section{Expression A-3}

As mentioned above, the expression (A-2) represents a 'neutral-approximation' where the influence of both magnetic $(B)$ and electric $(E)$ fields on particle trajectories is ignored. In other words the trajectories are those given for neutral particles. The influence of the $B$ and $E$ fields on the trajectories at different distances from the satellite (downstream) is given in detail in AL'PERT, 1976 and will not be discussed here. It is, however, worth mentioning that results regarding the influence of $E$ on the ion motion in a multi-component plasma were used to show that $(A-2)$ is valid for quite a number of practical situations in the ionosphere.
AL'PERT, YA. L.

Brace, L. H., Theis, R. F. and Dalgarno, A.

Brinton, H. C., Scott, L. R., Pharo, M. W. and Coulson, J. T. C.

Gurevich, A. V., Pitaevski, L. P. and SMIRNOVA, V. V.

Henderson, C. L. and SAmir, U.

PARKer, L. W.

PARKER, C. W.

SAMIr, and Willmore, A. P.

1965

SAMIR, U. and WRENN, G. L.

Samir, U., First, M., Maier, E. J. and TROY, B. E.

SAMir, U., Brace, L. H. and Brinton, H. C.

SAMIR, U., GORdON, R., BRACE, L. and THEIS, $\mathbf{R}$.

\section{REFERENCES}

1976 In: Handbuch der Physik, (Edited by S. FuugGe)

1973 Radio Sci. 8, 341.

1973 Radio Sci. 8, 323.

1970 Soviet Phys. Usp. 99, 595

1967 Planet. Space Sci. 15, 1499.

1976 Computation of collisionless steady-state plasma flow past a charged disc, Report, NASA CR144159, February.

Claculation of sheath and wake structure about a pillbox-shaped spacecraft in a flowing plasma, Proc. of the Spacecraft Charging Technology Conference, (Edited by C. P. PIKE and R. R. LOVELL), Joint Air Force-NASA Report AFGLTR-77-0051 and NASA TMX-73537, pp. 331360, February.

1969 Planet. Space Sci. 17, 693.

1975 J. atmos. terr. Phys. 37, 577.

1979a Geophys. Res. Lett. 6, 101.

$1979 \mathrm{~b} J$. geophys. Res. 84, 513. 